\title{
Habilidades de enfrentamento antecipatório para abstinência de substâncias: construção de um novo instrumento de medida
}

\author{
finticipatory Coping Skills for Substance flbstinence: Construction \\ of a new Measuring Scale \\ Habilidades de enfrentamiento anticipado para abstinencia de sustancias: \\ construcción de un nuevo instrumento de medida
}

\author{
Lucas Guimarães Cardoso de Sá, Zilda Aparecida Pereira del Prette ${ }^{* *}$ \\ ${ }^{*}$ Universidade Federal do Maranhão, Brasil. ${ }^{* *}$ Universidade Federal de São Carlos, Brasil.
}

Doi: http://dx.doi.org/10.12804/ap134.2.2016.09

\section{Resumo}

Habilidades de enfrentamento antecipatório são importantes para a manutenção da abstinência de álcool e outras drogas. Apesar disso, não se dispõe de instrumentos para avaliá-las em contexto brasileiro. Este artigo descreve as etapas iniciais da construção de um instrumento de medida para a avaliação desse repertório, o que envolveu várias etapas. Inicialmente, com base na literatura, em instrumentos semelhantes e em entrevistas, foram redigidos 92 itens, que contemplavam 14 classes de habilidades, definidas e exemplificadas no texto. A qualidade semântica e o conteúdo dos itens foram aferidos por juízes, resultando na eliminação de 24 deles. A nova versão passou por um pré-teste de funcionamento e estudo piloto com participantes em tratamento por abuso ou dependência de álcool ou outras drogas.
Novos ajustes foram realizados e o conjunto final, de 67 itens, recebeu o nome de Inventário de Habilidades de Enfrentamento Antecipatório para Abstinência de Álcool e Outras Drogas (IDHEA-AD). Discute-se cada uma das etapas e são apresentadas propostas para estudos futuros. Palavras-chave: enfrentamento; prevenção da recaída; abstinência; adicção; habilidades sociais.

\section{fibstract}

\begin{abstract}
Anticipatory coping skills are important for the maintenance of abstinence from alcohol and other drugs. Nevertheless, there are no instruments available to evaluate them in the Brazilian context. This paper describes the first developments of an instrument that permits the assessment of this repertoire, which involved several steps. Initially, based on the literature, on similar instruments
\end{abstract}

* Lucas Guimarães Cardoso de Sá, Departamento de Psicologia, Universidade Federal do Maranhão; ** Zilda Aparecida Pereira del Prette, Departamento de Psicologia, Universidade Federal de São Carlos. Esta pesquisa recebeu apoio da Fundação de Apoio à Pesquisa do Estado de São Paulo (FAPESP), Processo \#2010/07890-3.

A correspondência deste artigo deve ser endereçada para Lucas Guimarães Cardoso de Sá, Universidade Federal do Maranhão, Departamento de Psicologia, Avenida dos Portugueses, 1966, São Luís-Maranhão, Brasil. E-mail: lucas.gcs@gmail.com

Como citar este artigo: de Sá, L. G. \& del Prette, Z. A. (2016). Habilidades de enfrentamento antecipatório para abstinência de substâncias: construção de um novo instrumento de medida. Avances en Psicología Latinoamericana, 34(2), 351-363. doi: http://dx.doi.org/10.12804/apl34.2.2016.09 
and interviews, 92 items were written, including 14 skills classes, defined and exemplified in the text. Referees conducted the semantic and content analyses, and 24 items were excluded from the list. This new version was submitted to an operational pre test and a pilot study with participants treated for abuse or addiction to alcohol or other drugs. New adjustments were made and the final set of 67 items was named Anticipatory Coping Skills Inventory for Abstinence from Alcohol and Other Drugs (IDHEA-AD, as in Portuguese). Each one of those steps is discussed and proposals for future studies are presented. Keywords: coping; relapse prevention; abstinence; addiction; social skills.

\section{Resumen}

Las habilidades de enfrentamiento anticipado son importantes para mantener la abstinencia de alcohol y otras drogas. A pesar de eso, no se dispone de instrumentos para evaluarlas en el contexto brasilero. Este artículo describe las etapas iniciales de la construcción de un instrumento de medida para evaluar este repertorio, lo que incluyó varias etapas. Inicialmente, con base en la literatura, en instrumentos semejantes y en entrevistas, fueron redactados 92 ítems, que contemplaban 14 clases de habilidades, definidas y ejemplificadas en el texto. La calidad semántica y el contenido de los ítems fueron evaluados por jueces, de lo cual resultó la eliminación de 24 de ellos. La nueva versión pasó por un pretest de funcionamiento y por un estudio piloto con participantes en tratamiento por abuso o dependencia de alcohol $\mathrm{u}$ otras drogas. Nuevos ajustes fueron realizados y el conjunto final, de 67 ítems, recibió el nombre de Inventario de Habilidades de Enfrentamiento Anticipado para Abstinencia de Alcohol y Otras Drogas (IDHEA-AD). En el artículo se discuten cada una de las etapas y son presentadas propuestas para estudios futuros.

Palabras clave: enfrentamiento; prevención de recaída; abstinencia; adicción; habilidades sociales.

Variáveis diversas compõem um modelo explicativo multifatorial para o consumo abusivo ou dependente de uma substância. Uma delas, parte dos fatores psicológicos, são as habilidades de enfrentamento, um conjunto de estratégias cognitivas e comportamentais adaptativas, disponíveis no repertório do indivíduo para serem usadas em momentos específicos de proteção à abstinência (Donovan, 2009).

O retorno ao consumo compulsivo da substância após período de abstinência, conhecido como recaída, pode ser resultado de uma maneira desadaptativa de lidar com situações cotidianas de estresse, sejam em família, no trabalho, com amigos ou mesmo em breves interações com desconhecidos. O estresse, um desequilíbrio entre as exigências do ambiente e os recursos do indivíduo, levaria aqueles com repertório de habilidades de enfrentamento pouco elaborado a utilizarem a substância na tentativa de reestabelecer o equilíbrio (Monti, Kadden, Rohsenow, Cooney \& Abrams, 2005). Alguns estudos demonstram a relação entre estressores prévios e consumo de substâncias (Brown et al., 1990; Hassanbeigi, Askari, Hassanbeigi \& Pourmovahed, 2013; Saha, 2013).

Donovan (2009) descreve um subtipo específico de enfrentamento, denominado antecipatório, em que pensamentos e comportamentos são direcionados para a construção de um ambiente menos suscetível a situações estressoras ou para sua rápida e eficaz solução, antes que possam ocasionar fissura, vontade grande e imediata de usar a substância. Se não há fissura, as chances de uma recaída podem diminuir consideravelmente. Portanto, para manter a abstinência, seria importante que o indivíduo desenvolvesse um bom repertório de habilidades de enfrentamento antecipatório, como por exemplo, recusar assertivamente oferta de substâncias, controlar a raiva, fazer e receber elogios e lidar com críticas (Monti, Kadden, Rohsenow, Cooney \& Abrams, 2005).

Habilidades de enfrentamento são compostas em grande parte por habilidades sociais, estas definidas como classes de comportamentos sociais requeridas para completar de forma bem-sucedida 
uma determinada tarefa social (Del Prette \& Del Prette, 2008). Elas são fundamentais, apesar de não suficientes, para um comportamento ser avaliado como socialmente competente, ou seja, que resulte, conforme Del Prette e Del Prette (2012), na consecução dos objetivos de uma situação interpessoal, na manutenção ou melhora da relação com outras pessoas, na autoestima dos envolvidos na interação, na ampliação dos direitos humanos e na aprovação social. Embora não sejam sinônimos, já que as de enfrentamento são um construto mais amplo que inclui habilidades intrapessoais, são inegáveis a importância e o peso das habilidades sociais no processo na manutenção da abstinência. Para Rangé e Marlatt "o desenvolvimento de habilidades para enfrentamento efetivo precisa envolver habilidades sociais básicas, comportamentos assertivos e habilidades de confronto, que incluem a capacidade de identificar situações de risco, lidar com emoções e fazer reestruturações cognitivas" (2008, p. 92).

Alcançar critérios de competência social é uma condição fundamental para um indivíduo que deseja iniciar ou manter a abstinência. Como apontam Longabaugh e Morgenstein (1999), comportamentos pouco competentes socialmente poderiam gerar um conjunto de situações e sentimentos negativos, levando a uma menor eficiência no enfrentamento de situações adversas e dificultando as tentativas de manutenção da abstinência. Portanto, as habilidades sociais colaboram para a prevenção da recaída ao contribuírem para a competência social $\mathrm{e}$, por conseguinte, favorecerem o estabelecimento de vínculos responsáveis, estáveis e seguros (Sakiyama, Ribeiro \& Padin, 2012) e ampliarem a probabilidade de consecução de metas, como identificar e afastar-se das situações de risco e manter o autocontrole, além de melhorar a autoestima e a qualidade de suas relações com as demais pessoas (Villalba, 1995).

A afirmação de que o repertório de habilidades de enfrentamento está relacionado a aspectos da abstinência de substâncias psicoativas vem sendo encontrada na literatura desde a década de 1970 (Felicíssimo, Casela \& Ronzani, 2013; Longabaugh \& Morgenstein, 1999; Van Hasselt, Hersen \& Milliones, 1978). Contudo, mesmo após 40 anos, diversas características desta relação, sustentadas por evidências robustas, ainda não são conhecidas. Uma hipótese para explicar tais lacunas é que, ao longo dos anos, pouca prioridade foi dada a investigações psicométricas, raros foram os estudos que buscaram desenvolver ou aperfeiçoar instrumentos capazes de avaliar habilidades de enfrentamento especificamente na dependência de substâncias. Assim, o uso de medidas globais, tomadas com base em contextos inespecíficos, foi a alternativa disponível e provisoriamente justificável que, porém, acabou tornando-se o padrão estabelecido.

No entanto, a exemplo do que alertam Del Prette e Del Prette (2009) no caso das habilidades sociais, ao avaliar habilidades de enfrentamento parece ser relevante considerar sua característica situacional-cultural, ou seja, analisar os diferentes contextos, interlocutores, cultura e subcultura dos indivíduos que definem os comportamentos específicos efetivos e desejáveis. Em outras palavras, é possível supor que o enfrentamento na dependência de substâncias possui particularidades que não são captadas de forma confiável por medidas globais. Por isso a importância de instrumentos que sejam pautados por situações e comportamentos específicos desse contexto (Litt, Kadden \& Tennen, 2012). No entanto, os poucos instrumentos existentes para medir habilidades de enfrentamento específicas à dependência de substâncias não apresentam informações detalhadas sobre suas qualidades psicométricas (Humke \& Radnitz, 2005; Litman, Stapleton, Oppenheimer \& Peleg, 1983; Marijuana Treatment Project, 2004; Monti et al., 1993; Moos, 1992; Rohsenow et al., 2001; Rohsenow, Martin \& Monti, 2005). E mais, grande parte deles avalia diferentes tipos de habilidades de enfrentamento em conjunto, não exclusivamente habilidades antecipatórias. No Brasil, o problema parece ainda mais grave, pois não foi encontrado qualquer instrumento para a 
avaliação do enfrentamento no contexto do consumo de substâncias.

Um desafio metodológico para a construção de um instrumento específico, tal como antes defendido, está exatamente na identificação e construção dos itens que poderiam ser tomados como representativos do construto "enfrentamento antecipatório". Trata-se de uma etapa que requer um conjunto de técnicas, nem sempre descritas e explicitadas na literatura psicométrica. Pasquali (2010) propõe um modelo orientado e sequencial para a construção de instrumentos psicológicos, dividindo o processo em três grandes polos. No primeiro deles, teórico, a literatura deve subsidiar a operacionalização do construto em itens. No segundo, experimental, o instrumento construído deve ser testado quanto a procedimentos de aplicação. Por fim, no terceiro polo, o analítico, são estabelecidos procedimentos de análise estatística que indicarão evidências de validade e fidedignidade.

Considerando o papel fundamental do enfrentamento antecipatório, por ser o primeiro na linha temporal de prevenção da recaída, o objetivo deste texto é descrever os polos teórico e experimental do processo de construção de um instrumento para avaliar habilidades de enfrentamento antecipatório, requeridas na manutenção da abstinência de álcool e outras drogas. No polo teórico, é apresentada primeiramente a operacionalização do construto por meio de itens e em seguida a análise de construto e semântica destes itens. No polo experimental são apresentados os resultados e considerações sobre as aplicações testes do instrumento. Para tornar o texto mais didático, uma vez que diversos procedimentos compõem todo o processo de construção do instrumento, o polo teórico será apresentado em duas partes, que somadas ao polo experimental, formam três etapas. Cada uma será descrita separadamente, com sua organização própria, mas devem ser analisadas sempre como articuladas sequencialmente entre si. Os cuidados éticos foram respeitados em todas as etapas.

\section{Polo teórico}

Etapa 1. Operacionalização do construto: elaboração de itens para avaliar habilidades de enfrentamento na manutenção da abstinência de álcool e outras drogas

Essa etapa constou de três procedimentos: revisão de literatura, revisão de instrumentos existentes e entrevistas. A revisão da literatura tem a função de organizar e direcionar a construção dos itens. Somente a partir de uma base teórica sólida é que serão definidos os tipos e as características dos comportamentos que deverão representar o construto no instrumento (Pasquali, 2010). A revisão de instrumentos existentes envolve, conforme Pasquali “... inspirar em itens que compõem outros instrumentos disponíveis no mercado e que medem o mesmo construto no qual estou interessado" (2010, p. 176). Os itens não são copiados, mas esta análise deve garantir, com base na literatura, que o novo instrumento contenha os comportamentos já avaliados por outras escalas e inclua outros, anteriormente faltantes. Por fim, as entrevistas, por serem dirigidas aos representantes da população estudada, oferecem um grande número de respostas que representam diretamente o construto que se pretende avaliar. Mais que isso, permitem que os itens englobem situações e detalhes da cultura que dificilmente seriam alcançados por outro procedimento. Em certos casos, palavras e expressões típicas podem ser incluídas no instrumento, evitando uma redação que não seria compreensível ou que pudesse ser percebida como estranha. O método utilizado para cada um dos três procedimentos está descrito a seguir e ao final dessa seção são apresentados e discutidos os resultados obtidos.

\section{Revisão de literatura}

Procedimento. A seleção de habilidades de enfrentamento descritas em literatura foi iniciada 
com a análise de três publicações de referência, que descrevem habilidades de enfrentamento para o tratamento do alcoolismo (Monti et al., 2005), para o tratamento de usuários de cocaína (Carroll, 1998) e para o tratamento de usuários de maconha (Jungerman \& Zanelatto, 2007). Os modelos propostos são semelhantes, sugerindo que o enfrentamento não se diferencia conforme o tipo de substância utilizada. Tal hipótese parece amparada por Donovan (2009), que não apresenta habilidades de enfrentamento distintas para diferentes substâncias. Também foram analisados 31 artigos científicos, definidos após revisão sistemática de literatura em plataformas de busca. Todas as habilidades investigadas ou, citadas como importantes, para o enfrentamento na manutenção da abstinência de álcool ou outras drogas foram selecionadas. A lista de artigos e detalhes sobre este procedimento podem ser consultados em Sá (2013).

\section{Revisão de instrumentos existentes}

Procedimento. Foram selecionados sete instrumentos comumente utilizados para avaliar o enfrentamento nos transtornos relacionados ao uso de substâncias: CBI (Coping Behaviours Inventory), de Litman et al. (1983); CRI (Coping Response Inventory), de Moos (1992); USS (Urge-Specific Strategies Questionaire), de Monti et al. (1993) e Rohsenow et al. (2001); CSS (The Coping Strategy Scale), de Marijuana Treatment Project (2004); USS-C (Urge Specific Strategies Questionnaire-Cocaine Version) e GCS-C (General Change Strategies-Cocaine Version), ambos de Rohsenow et al. (2005); AACRI (The Alcohol Abuse Coping Response Inventory), de Humke e Radnitz (2005). Todos os itens foram analisados e aqueles que representavam habilidades de enfrentamento antecipatório foram destacados, de maneira a assegurar que os comportamentos por eles avaliados fossem reproduzidos nos itens construídos para o novo instrumento desenvolvido.

\section{Entrevistas}

Participantes. Os participantes foram 35 pessoas que responderam a uma entrevista sobre as habilidades de enfrentamento antecipatórias necessárias no processo de manutenção da abstinência de álcool ou outras drogas. Todos tiveram problemas relacionados ao consumo de substâncias e procuraram tratamento voluntário para iniciar ou manter a abstinência. Para participar era necessário relatar estar sem usar a substância há pelo menos 30 dias. O menor tempo de abstinência foi de um mês e o maior de 23 anos, com mediana de seis meses. Desses, 97\% eram homens, com média de idade de 37.4 anos. A maior parte $(n=23)$ relatou estar em tratamento por problemas relacionados principalmente ao consumo de crack, outros $11 \mathrm{de}$ álcool e um de maconha.

Instrumento. Uma versão adaptada da "Entrevista sobre Gatilhos do Álcool e Estratégias Específicas às Fissuras", contida em sua forma original em Monti et al. (2005, pp.164-166). As adaptações foram realizadas com o objetivo de se conseguir respostas de habilidades de enfrentamento antecipatório. Para aqueles em manutenção da abstinência de outras drogas, as palavras álcool e alcoolismo foram substituídas por droga e usar droga.

Procedimentos. As respostas ao roteiro de entrevista foram gravadas em áudio e posteriormente foi feita a transcrição das habilidades relatadas pelos participantes.

\section{Resultados e discussão}

A revisão da literatura proporcionou a elaboração de 16 categorias de habilidades de enfrentamento antecipatório. Já as entrevistas resultaram em outras 18 categorias, provenientes do agrupamento por conteúdo de 413 respostas oferecidas pelos entrevistados. Após esta etapa foram realizados 


\section{Lucas Guimarães Cardoso de Sá, Zilda Aparecida Pereira del Prette}

sucessivos agrupamentos por semelhança de conteúdo das categorias obtidas, até alcançar uma lista final contendo 14 classes distintas de habilidades de enfrentamento antecipatório (tabela 1). Com base na descrição feita para cada uma, foram redigidos 92 itens que poderiam representar o construto.

Os itens foram redigidos contendo a descrição de uma situação comum ao contexto do consumo de substâncias e uma estratégia de ação diretamente relacionada a ela, como pode ser visto na tabela 1 . Os participantes são solicitados a responder a frequência com que desempenham a estratégia, no momento atual da avaliação, usando uma escala de quatro pontos de resposta (nunca, poucas vezes, muitas vezes, sempre). $\mathrm{O}$ instrumento foi nomeado Inventário de Habilidades de Enfrentamento Antecipatório para a Abstinência de Álcool e Outras Drogas (IDHEA-AD).

Algumas habilidades de enfrentamento antecipatório envolvem evitar o engajamento em comportamentos desadaptativos. No entanto, avaliar a evitação de algo negativo envolve dupla negação, o que torna o item confuso e pode gerar sérias

Tabela 1

Descrição das classes de habilidades de enfrentamento antecipatório e exemplos de itens elaborados para representá-las

Classe de habilidade
Habilidades para recusar oferta de bebida ou droga. Desta-
ca-se nas respostas que muitas vezes não é possível apenas
ignorar a oferta, é necessário recusar de maneira assertiva.
Habilidades de planejamento. Envolvem a avaliação prévia
de potenciais situações de risco e o direcionamento de pensa-
mentos e comportamentos para enfrentá-los.
Habilidade para receber elogios, especialmente sobre bene-
fícios resultantes da abstinência. Em menor escala, também
para fazer elogios gerais.
Habilidades para responder adequadamente a pessoas que
fazem propaganda da substância, dizendo que ela é boa ou
traz efeitos prazerosos.

Habilidades para solicitar mudanças de comportamentos que trazem risco à manutenção da abstinência.

Habilidades para ouvir e responder adequadamente a brincadeiras em relação à abstinência, evitando o surgimento de irritação, raiva ou tristeza.

Habilidades para ouvir conselhos e dicas sobre como manter a abstinência, sem oferecer reações indesejadas.

Habilidades de autocontrole emocional especialmente em situações de raiva ou irritação, que se não forem manejadas adequadamente podem se tornar gatilho para o uso da substância.

Habilidades de resolução de problemas gerais, mas especialmente os de relacionamento com familiares.

Habilidades para lidar com humores negativos (como frustração, tristeza, depressão, tensão, preocupação, solidão), mas também positivos (como euforia e felicidade extrema).
Exemplo de item construído para representar a classe

Se alguém me oferece (nome da substância), sugiro fazer outra coisa, mais saudável.

Se preciso ir à um local em que haverá (nome da substância), faço um roteiro planejando tudo o que preciso fazer para não recair.

Se recebo elogios sobre as mudanças positivas resultantes da abstinência, digo para a pessoa que fiquei feliz por ela ter percebido as mudanças.

Se alguém me diz que (nome da substância) é algo bom, respondo que para mim nenhuma substância desse tipo faz bem.

Se uma pessoa vem usar (nome da substância) na minha frente, eu peço para ela parar.

Se um conhecido faz piada com o fato de que eu parei de usar (nome da substância), procuro conversar com uma pessoa de confiança para me aliviar.

Tenho dificuldade de ouvir com atenção quando alguém me dá conselhos sobre como não usar (nome da substância).*

Se estou com raiva, penso em frases que podem me acalmar (por exemplo, "fica frio", "vai com calma").

Se eu tenho um desentendimento com um familiar, tento fazer um acordo com a pessoa para resolver o problema.

Se estou me sentindo triste, expresso esse sentimento para alguém de minha confiança. 
Classe de habilidade

Habilidades para estabelecer bom relacionamento com familiares. Envolve tanto apresentar quanto responder a comportamentos como os de civilidade, expressão de sentimentos positivos e de ajuda.

Habilidades para receber críticas justas, especialmente em relação a comportamentos relacionados ao uso de substâncias. Envolve habilidades para assumir erros, pedir desculpas e oferecer a possibilidade de mudar o comportamento.

Habilidades para receber criticas injustas ou mal executadas. Envolve principalmente lidar com desconfiança sobre o uso de substância e cobrança por uma recuperação imediata e definitiva.

Habilidades sociais gerais, mas especialmente de conversação e desenvoltura social.
Exemplo de item construído para representar a classe

Se eu recebo carinho/afeto de alguém, por estar sem usar (nome da substância), sinto vergonha de devolver carinho/ afeto para a pessoa.*

Acho dificil pedir desculpas quando alguém mostra algo de errado que eu realmente fiz.*

Se um familiar desconfia injustamente que eu usei (nome da substância), explico calmamente que a situação agora é diferente.

Se quero fazer amizade com uma pessoa, inicio uma conversa sem ter dificuldade.

*Exemplos de itens elaborados com sentido negativo, visando diminuir o impacto da desejabilidade social.

confusões ao respondente. Por isso, em vez de avaliar a frequência de evitação de um comportamento desadaptativo, alguns itens foram elaborados para avaliar a frequência de dificuldade para desempenhar o comportamento adaptativo. Por exemplo, um item como "Evito mentir quando erro" seria redigido como "Tenho dificuldade em dizer a verdade quando erro". Este procedimento é semelhante ao adotado por Del Prette e Del Prette (2001). Além de possibilitar respostas mais claras, evita que o instrumento contenha apenas comportamentos desejáveis, o que poderia gerar maiores efeitos de desejabilidade social ou automatismo de respostas como, por exemplo, assinalar as opções das extremidades sem a leitura atenta de cada um deles. Isto poderia afetar a variabilidade dos dados obtidos e, consequentemente, a precisão do instrumento. No momento da interpretação dos resultados, todos os itens elaborados desta maneira são pontuados de forma inversa, de forma a se obter sempre uma medida uniforme, com sentido positivo, das habilidades do indivíduo.

\section{Etapa 2. Análise da qualidade dos itens}

A simples operacionalização do construto, como apresentado na etapa anterior, não garante que o instrumento meça com qualidade o que se pretende. Há diversas maneiras de testar a adequação do instrumento. Ainda dentro do polo teórico, uma maneira comum de se fazer isso, de acordo com Pasquali (2010), é pedir que outras pessoas, devidamente qualificadas, avaliem cada um dos itens, tanto em relação à representação do construto (análise de conteúdo) quanto a aspectos relacionados à forma (análise semântica). O IDHEA-AD foi submetido às duas análises, conforme apresentado a seguir.

\section{Análise de conteúdo}

Participantes. Participaram 14 psicólogos e quatro assistentes sociais, sem experiência em Psicometria, mas com média de oito anos de experiência acadêmica ou profissional em transtornos relacionados ao uso de substâncias,

Instrumento. A versão inicial do Inventário de Habilidades de Enfrentamento Antecipatório para a Abstinência de Álcool e Outras Drogas (IDHEA-AD), com 92 itens.

Procedimentos. Os participantes assumiram a função de juízes e avaliaram se o conteúdo de cada um dos itens representava uma habilidade 
de enfrentamento antecipatório. Para essa tarefa indicaram apenas "sim" ou "não". Em seguida, aqueles itens com resposta positiva também foram analisados quanto à sua importância para o processo de enfrentamento e manutenção da abstinência. Nesse caso, os juízes anotaram um número entre zero (nada importante) e cinco (totalmente importante). Para ser mantido no instrumento o item deveria ter no mínimo $70 \%$ de concordância em relação à representação do construto e ao menos 60\% na avaliação de importância.

\section{Análise semântica}

Participantes. A amostra foi composta por 12 pessoas com nível superior de escolaridade, sem experiência na área da dependência de substâncias.

Instrumento. A versão do IDHEA-AD posterior à análise de conteúdo.

Procedimentos. Os participantes avaliaram se as frases dos itens estavam bem redigidas, organizadas, se havia palavras de difícil compreensão, sentido dúbio ou qualquer outra imperfeição. Ressaltava-se aos juízes que pessoas com pouco estudo deveriam ser capazes de responder as questões do instrumento. Além de indicar possíveis problemas, os juízes poderiam sugerir alterações ou mesmo reescrever o item, de modo a torná-lo mais compreensível. Uma última tarefa solicitada foi observar se dois ou mais itens eram semelhantes e poderiam ser representados por apenas um.

\section{Resultados e discussão}

Dos 92 itens construídos inicialmente para operacionalizar o construto, nove não atingiram o critério estabelecido para a representação adequada do construto e foram eliminados. De 83 restantes, 12 não alcançaram o critério mínimo estabelecido para a importância e também foram excluídos. Com isso, o IDHEA-AD passou a conter
71 questões. Na análise semântica, as principais sugestões oferecidas pelos juízes tiveram o objetivo de deixar as questões mais compreensíveis a pessoas de pouca escolaridade. Seguindo estas recomendações, alguns itens foram reescritos da maneira mais simples possível, adequando-se mais à linguagem falada que à culta. A avaliação dos árbitros foi que a compreensão pelos respondentes é mais importante que a correção formal da frase. Além disso, houve indicação de que três pares de itens estavam avaliando comportamentos muito semelhantes e poderiam ser fundidos, obtendo-se o mesmo resultado. As sugestões foram aceitas e o instrumento passou a conter 68 questões.

Os resultados desta etapa trouxeram importantes modificações na estrutura do instrumento, pois com os critérios estabelecidos, foram excluídos 24 dos itens redigidos inicialmente. Destaca-se, porém, que a exclusão proposta pelos juízes não foi aleatória, mas seguiu um padrão, no qual comportamentos de evitação, como por exemplo "isolar-se após desentendimento" ou "calar-se após uma crítica injusta recebida", não foram avaliados como verdadeiras habilidades de enfrentamento ou foram considerados pouco relevantes para a prevenção da recaída. O padrão adotado pelos juízes consultados neste estudo faz parte de uma discussão ainda em aberto na literatura. Enquanto autores como Armeli, Dehart, Tennen, Todd e Affleck (2007), Farabee et al. (2013) e Litt, Kadden, Cooney e Kabela (2003) indicam pouca evidência de que estilo de enfrentamento de evitação está associado a problemas relacionados ao consumo de substâncias, outros como Forys, McKellar e Moos (2007), Monti et al. (2005), Moser e Annis (1996) e Wunschel, Rohsenow, Norcross e Monti (1993) indicam o contrário. Como não há um consenso, optou-se por apoiar o parecer dos juízes e considerar que habilidades de evitação, embora possam ser úteis no início da abstinência, não representam um real enfrentamento das situações e não colaboram para a abstinência em longo prazo. Assim, se não há aprendizado de habilidades mais ativas, entende-se que as chances 
de recaída podem aumentar consideravelmente. Por esse motivo, o IDHEA-AD contém habilidades predominantemente ativas.

\section{Polo experimental}

\section{Etapa 3. Aplicações testes do IDHEA-AD}

Cumpridos os procedimentos do polo teórico, deve-se avançar para os seguintes, relacionados ao polo experimental. Os procedimentos empíricos estão relacionados a aspectos mais práticos de utilização do instrumento. Envolvem a relação entre respondentes, com suas características pré-determinadas pelos objetivos do instrumento, e o planejamento de aplicação, como tipos de aplicação, instruções, formato das respostas, tempo de resposta, entre outros. Para o IDHEA-AD, foram adotados dois procedimentos. O primeiro, chamado de aplicação inicial, foi realizado para verificar possíveis problemas adicionais de clareza e compreensão das questões, não perceptíveis aos juízes da etapa anterior, mas perceptíveis para indivíduos semelhantes à população à qual se destina o instrumento. O segundo, chamado de aplicação piloto, teve o mesmo objetivo, mas foi realizado com amostra real da população alvo do instrumento. Os dois são descritos e analisados a seguir.

\section{Aplicação inicial}

Participantes. Participaram três homens e três mulheres, sem diagnóstico de abuso ou dependência de substâncias, com níveis distintos e crescentes de escolaridade, desde ensino fundamental incompleto até ensino superior completo.

Instrumento. A versão IDHEA-AD com 68 itens.

Procedimentos. Foi simulada uma aplicação do instrumento. Foi marcado o tempo gasto entre a entrega de uma versão impressa do IDHEA-AD e sua devolução, totalmente preenchida. O pesquisador acompanhou o preenchimento do instrumento, observando todo o processo de resposta. Quando o tempo de resposta para um item era maior que a média dos demais ou se o próprio participante solicitava esclarecimento, pausava-se o relógio, as dúvidas da questão eram discutidas, anotadas e uma redação mais clara era elaborada. Após tal procedimento, o relógio era novamente acionado e a aplicação continuava.

\section{Aplicação piloto}

Participantes. A amostra foi composta por 91 pessoas, sendo $92.3 \%$ homens, média de idade de 39 anos, 63.8\% com escolaridade básica, 26.4\% com escolaridade média e $9.9 \%$ com escolaridade superior. A maior parte, 54\%, era de usuários exclusivos de álcool e o restante de múltiplas substâncias, mas que relatavam problemas relacionados essencialmente ao consumo de crack. Todos estavam sob tratamento em serviços públicos ou filantrópicos de atenção à dependentes de substâncias. Quanto ao tipo de intervenção, $49.5 \%$ estavam em regime intensivo, permanecendo no local de tratamento 24 horas por dia. Outros $48.4 \%$ estavam em regime semi-intensivo, nunca ultrapassando doze horas por dia e cinco dias por semana. Por fim, 2.2\% freqüentavam grupos de ajuda mútua, em reuniões semanais. O menor tempo de abstinência relatado foi de três dias e o maior de 12 anos, com mediana de 2.5 meses.

Instrumento. O IDHEA-AD, na versão posterior às modificações pontuais efetuadas na aplicação inicial (ver seção Resultados e Discussão desta Etapa 3).

Procedimentos. Três formas de aplicação foram utilizadas: $45.1 \%$ dos participantes passaram por leitura oral individual, em que o pesquisador lia as perguntas e anotava as respostas; $35.1 \%$ por autoaplicação, em que o próprio participante lia 
e respondia as questões; e $19.8 \%$ por leitura oral em grupo, na qual o pesquisador lia as perguntas em voz alta para grupos que variaram de três a seis componentes. Em todas elas, foi verificada a compreensão das questões pelos respondentes e, quando necessário, foram esclarecidas dúvidas, solicitadas sugestões e realizadas alterações na redação dos itens. As respostas obtidas foram transformadas em códigos numéricos e digitadas em uma planilha da versão 18 do programa de computador $P A S W$ Statistics. Para cada item calculou-se a média, o desvio padrão, a porcentagem com que cada ponto da escala de resposta foi assinalado e valores de assimetria e curtose. Estes dados podem ser consultados em Sá (2013).

\section{Resultados e discussão}

No procedimento de aplicação inicial, o tempo total de resposta ao IDHEA-AD variou entre 17 e 41 minutos. Como esperado, menor nível de escolaridade parece implicar em maior tempo de resposta ao instrumento. Apesar disso, essa variável não pareceu ter interferido no entendimento das questões. Independentemente do nível de escolaridade, nenhuma frase ou palavra foi apontada como de difícil compreensão. Alterações pontuais foram efetuadas, principalmente em relação à inversão da ordem de sentenças ou de palavras específicas dentro de um item, de forma a deixá-lo mais claro.

Os resultados da aplicação piloto mostraram indícios de adequação do instrumento, com itens com médias e desvios padrões plausíveis e boa distribuição das respostas pelos quatro pontos da escala. Isso também foi confirmado pelos valores próximos $\mathrm{a} \pm 1.00$ para a assimetria $\mathrm{e} \pm 3.00$ para curtose, conforme critérios para interpretação da qualidade da distribuição propostos por Hair Jr., Babin, Money e Samouel (2005). Apenas um item foi excluído, por apresentar tanto assimetria quanto curtose muito acima dos valores de referência.

Para habilidades relacionadas à civilidade e à expressão de sentimentos positivos, os valores foram limítrofes. Aparentemente elas ensejam um componente de desejabilidade social no qual o indivíduo tende a assinalar os pontos mais elevados da escala de resposta, indicando o desempenho mais freqüente da habilidade. Uma hipótese para explicar este comportamento é que assumir o contrário pode ser algo que não é bem visto socialmente, $o$ que gera expectativa de conseqüências negativas. Para diminuir o impacto da desejabilidade social, optou-se por reescrever quatro desses itens. Assim, em vez de avaliar a frequência de desempenho de habilidades de civilidade e expressão de afeto positivo, passaram a avaliar a dificuldade para desempenhá-las, com o respondente tendo que assinalar a frequência com que faz o inverso do socialmente esperado. Por exemplo, um item que antes era descrito como "Expresso afeto a meus familiares", passou a ser apresentado como "Tenho dificuldade de expressar afeto a meus familiares". O esperado é que essa modificação possibilite uma avaliação menos automática e gere respostas mais precisas. No momento da apuração, itens desse tipo devem ter seus valores invertidos para uniformizar os resultados e facilitar a interpretação.

\section{Considerações finais}

A partir de lacunas teóricas e práticas existentes, surgiu a necessidade de desenvolver um novo instrumento de avaliação das habilidades de enfrentamento que fosse específico para o contexto do abuso e dependência de substâncias e considerasse as particularidades da cultura brasileira. Os primeiros esforços nesse sentido, apresentados neste artigo, resultaram no IDHEA-AD, um instrumento com 67 itens, sendo 53 avaliando a frequência de desempenho de comportamentos adaptativos e 14 a dificuldade para desempenhá-los, em diferentes situações, contextos e com interlocutores diversos.

O surgimento de uma nova ferramenta de medida parece representar um importante avanço. Um instrumento como esse pode colaborar para uma melhor avaliação dos padrões cognitivos e de 
comportamentos sociais associados à abstinência $\mathrm{e}$ ao consumo de substâncias psicoativas, o que pode, por sua vez, subsidiar estratégias de intervenção mais eficazes. Ele permitirá, por exemplo, identificar habilidades relevantes e produzir indicadores mais precisos e válidos para avaliar a efetividade das intervenções.

As regras relacionadas ao desenvolvimento de instrumentos psicológicos e sua utilização prática são bastante rígidas atualmente. O Conselho Federal de Psicologia, fundamentado em diretrizes internacionais, propostas pela International Test Commission, pela American Educational Research Association, American Psychological Association \& National Council on Measurement in Education e pela Canadian Psychological Association, exige, desde sua resolução número dois do ano de 2003, uma série de requisitos para que um instrumento seja reconhecido pela comunidade científica e profissional (Conselho Federal de Psicologia, 2010). É necessário apresentar diversas evidências de validade, dados relacionados às propriedades dos itens, à precisão do instrumento e um sistema de correção baseado em amostras brasileiras da população ao qual se destina o instrumento, permitindo a interpretação dos resultados obtidos por um indivíduo.

Dessa maneira, como apenas os polos teórico e experimental foram contemplados até o momento, é necessário prosseguir com o desenvolvimento do IDHEA-AD, de forma a abranger também o polo analítico. O prosseguimento das investigações sobre a qualidade do IDHEA-AD, mais que uma recomendação, constitui uma necessidade. Portanto, a próxima etapa deverá envolver a realização de uma Análise Fatorial Exploratória, que mostre evidências de validade baseada na estrutura interna do instrumento. Em seguida, é importante investigar a estrutura obtida, por meio de Análise Fatorial Confirmatória. Ao mesmo tempo devem ser extraídos indicadores de precisão, como os alfas de Cronbach e confiabilidades compostas. Com este suporte, outras evidências de validade, baseadas nas relações com variáveis externas, e outros indicadores de fidedignidade, como estabilidade temporal, poderão ser buscados. Por fim, o estabelecimento de normas de interpretação encerraria um ciclo inicial de análises, permitindo a utilização mais segura do instrumento na prática profissional.

\section{Referências}

Armeli, S., Dehart, T., Tennen, H., Todd, M., \& Affleck, G. (2007). Daily interpersonal stress and the stressor-vulnerability model of alcohol use. Journal of Social and Clinical Psychology, 26(8), 896-921. doi: 10.1521/jscp.2007.26.8.896

Brown, S. A., Vik, P. W., McQuaid, J. R., Patterson, T. L., Irwin, M. R., \& Grant, I. (1990). Severity of psychosocial stress and outcome of alcoholism treatment. Journal of Abnormal Psychology, 99, 344-348. doi: 10.1037//0021-843X.99.4.344

Carroll, K. M. (1998). A cognitive-behavioral approach: Treating cocaine addiction. Rockville: National Institute on Drug Abuse.

Conselho Federal de Psicologia. (2010). Avaliação psicológica: diretrizes na regulamentação da profissão. Brasília: CFP.

Del Prette, Z. A. P. \& Del Prette, A. (2001). Inventário de Habilidades Sociais (IHS-Del Prette): Manual de aplicação, apuração e interpretação. São Paulo: Casa do Psicólogo.

Del Prette, Z. A. P. \& Del Prette, A. (2008). Psicologia das habilidades sociais: Terapia, educação e trabalho (5a ed.). Petrópolis: Vozes.

Del Prette, Z. A. P. \& Del Prette, A. (2009). Avaliação de habilidades sociais: bases conceituais, instrumentos e procedimentos. Em Z. A. P. del Prette \& A. del Prette (Orgs.), Psicologia das habilidades sociais: diversidade teórica e suas implicações (pp. 189-231). Petrópolis: Vozes.

Del Prette, Z. A. P. \& Del Prette, A. (2012). Social skills and behavior analysis: Historical proximity and new issues. Perspectivas em Análise do Comportamento, 1(2), 104-115. 
Donovan, D. M. (2009). Avaliação dos comportamentos dependentes na prevenção da recaída. Em Donovan \& Marlatt (Orgs.), Avaliação dos comportamentos dependentes (pp. 1-50). São Paulo: Roca.

Farabee, D., McCann, M., Brecht, M., Cousins, S. J., Antonini, V. P., Lee, A. B., et al. (2013). An analysis of relapse prevention factors and their ability to predict sustained abstinence following treatment completion. The American Journal on Addictions, 22(3), 206-211. doi: 10.1111/j.15210391.2012.00328.x

Felicíssimo, F. B., Casela, A. L. M., \& Ronzani, T. M. (2013). Habilidades sociais e alcoolismo: uma revisão da literatura. Psicologia em Estudo, 18(1), 137-145.

Forys, K., McKellar, J., \& Moos, R. (2007). Participation in specific treatment components predicts alcohol-specific and general coping skills. Addictive Behaviors, 32, 1669-1680. doi: 10.1016/j.addbeh.2006.11.023

Hair Jr., J. F., Babin, B., Money, A. H., \& Samouel, P. (2005). Fundamentos de métodos de pesquisa em administração. Porto Alegre: Bookman.

Hassanbeigi, A., Askari, J., Hassanbeigi, D., \& Pourmovahed (2013). The relashionship between stress and addiction. Procedia-Social and Behavioral Sicences, 84, 1333-1340. doi: 10.1016/j. sbspro.2013.06.752

Humke, C. \& Radnitz, C. L. (2005). An instrument for assessing coping with temptation: psychometric properties of the alcohol abuse coping response inventory. Substance Use and Misuse, 40 (1), 37-62. doi: 10.1081/JA-200030493

Jungerman, F. S. \& Zanelatto, N. A. (2007). Tratamento psicológico do usuário de maconha e seus familiares: um manual para terapeutas. São Paulo: Roca.

Litt, M. D., Kadden, R. M., \& Tennen, H. (2012). The nature of coping in treatment for marijuana dependende: Latent structure and validation of the coping strategies scale. Psychology of Addictive Behaviors, 26(4), 791-800.
Litt, M. D., Kadden, R. M., Cooney, N. L., \& Kabela, E. (2003). Coping skills and treatment outcomes in cognitive-behavioral and international group therapy for alcoolism. Journal of Consulting and Clinical Psychology, 71, 118-128.

Litman, G. K., Stapleton, J., Oppenheimer, A. N., \& Peleg, M. (1983). An instrument for measuring coping behaviors in hospitalized alcoholics: Implications for relapse prevention treatment. British Journal of Addiction, 78, 269-276. doi: 10.1111/j.1360-0443.1983.tb02511.x

Longabaugh, R. \& Morgenstein, J. (1999). Cognitive-Behavioral coping skills therapy for alcohol dependence: Current status and future directions. Alcohol Research \& Health, 23(2), 78-85.

Marijuana Treatment Project Research Group. (2004). Brief treatments for cannabis dependence: Findings from a randomized multisite trial. Journal of Consulting and Clinical Psychology, 72, 455-466.

Monti, P.M., Kadden, R.M., Rohsenow, D.J., Cooney, N.L., \& Abrams, D.B. (2005). Tratando a dependência de álcool: um guia de treinamento das habilidades de enfrentamento. São Paulo: Roca.

Monti, P.M., Rohsenow, D. J., Rubonis, A.V., Niaura, R. S., Sirota, A. D., Colby, S. M., et al. (1993). Cue exposure with coping skills treatment for male alcoholics: A preliminary investigation. Journal of Consulting and Clinical Psychology, 61, 1011-1019. doi: 10.1037//0022006X.61.6.1011

Moos, R. H. (1992). Coping Response Inventory Adult form. Palo Alto: Center for Health Care Evaluation, Stanford University and Department of Veterans Affairs Medical Center.

Moser, A. E. \& Annis, H. M. (1996). The role of coping in relapse crisis outcome: A prospective study of treated alcoholics. Addiction, 91, 1101-1113. doi: 10.1046/j.1360-0443.1996.91811013.x

Pasquali, L. (2010). Instrumentação psicológica: Fundamentos e práticas. Porto Alegre: Artmed. 
Rangé, B. \& Marlatt, G. A. (2008). Terapia cognitivo-comportamental de transtornos de abuso de álcool e drogas. Revista Brasileira de Psiquiatria, 30, 88-95.

Rohsenow, D. J., Martin, R. A., \& Monti, P. M. (2005). Urge-specific and lifestyle coping strategies of cocaine abusers: Relationships to treatment outcomes. Drug and Alcohol Dependence, 78, 211219. doi: 10.1016/j.drugalcdep.2005.03.001

Rohsenow, D. J., Monti, P. M., Rubonis, A. V., Gulliver, S. B., Colby, S. M., Binkoff, J. A. et al. (2001). Cue exposure with coping skills training and communication skills training for alcohol dependence: Six and twelve month outcomes. Addiction, 96, 1161-1174.

Sá, L. G. C. (2013). Propriedades psicométricas do Inventário de Habilidades de Enfrentamento para a Abstinência de Álcool e Outras Drogas (IDHEA-AD) (Tese de doutorado não publicada). Universidade Federal de São Carlos, São Carlos, Brasil.
Saha, A. (2013). A study on relapse prevention in cases of alcohol dependence syndrome. American Journal of Life Sciences, 1(4), 184-188.

Sakiyama, H.M.T., Ribeiro, M. \& Padin, M.F.R. (2012). Prevenção de recaída e habilidades sociais. Em M. Ribeiro \& R. Laranjeira (Orgs.), $O$ tratamento do usuário de crack (pp. 337-350). Porto Alegre: Artmed.

Van Hasselt, V. B., Hersen, M., \& Milliones, J. (1978). Social skills training in alcoholics and drug addicts: A review. Addictive Behaviors, 3, 221233. doi: 10.1016/0306-4603(78)90023-0

Villalba, E.A. (1995). Proyecto de entrenamiento en habilidades socials com toxicómanos. Em F.G. Rodríguez, J.M.L. Rubio \& L.J. Expósito (Orgs.), Habilidades sociales y salud (pp.127132). Madri: Biblioteca Eudema.

Wunschel, S. M., Rohsenow, D. J., Norcross, J. C., \& Monti, P. M. (1993). Coping strategies and the maintenance of change after inpatient alcoholism treatment. Social Work Research and Abstracts, 29, 18-22. doi: 10.1093/swra/29.4.18

\section{Recebido: 27 de março de 2014 Aprovado: 9 de junho de 2015}


